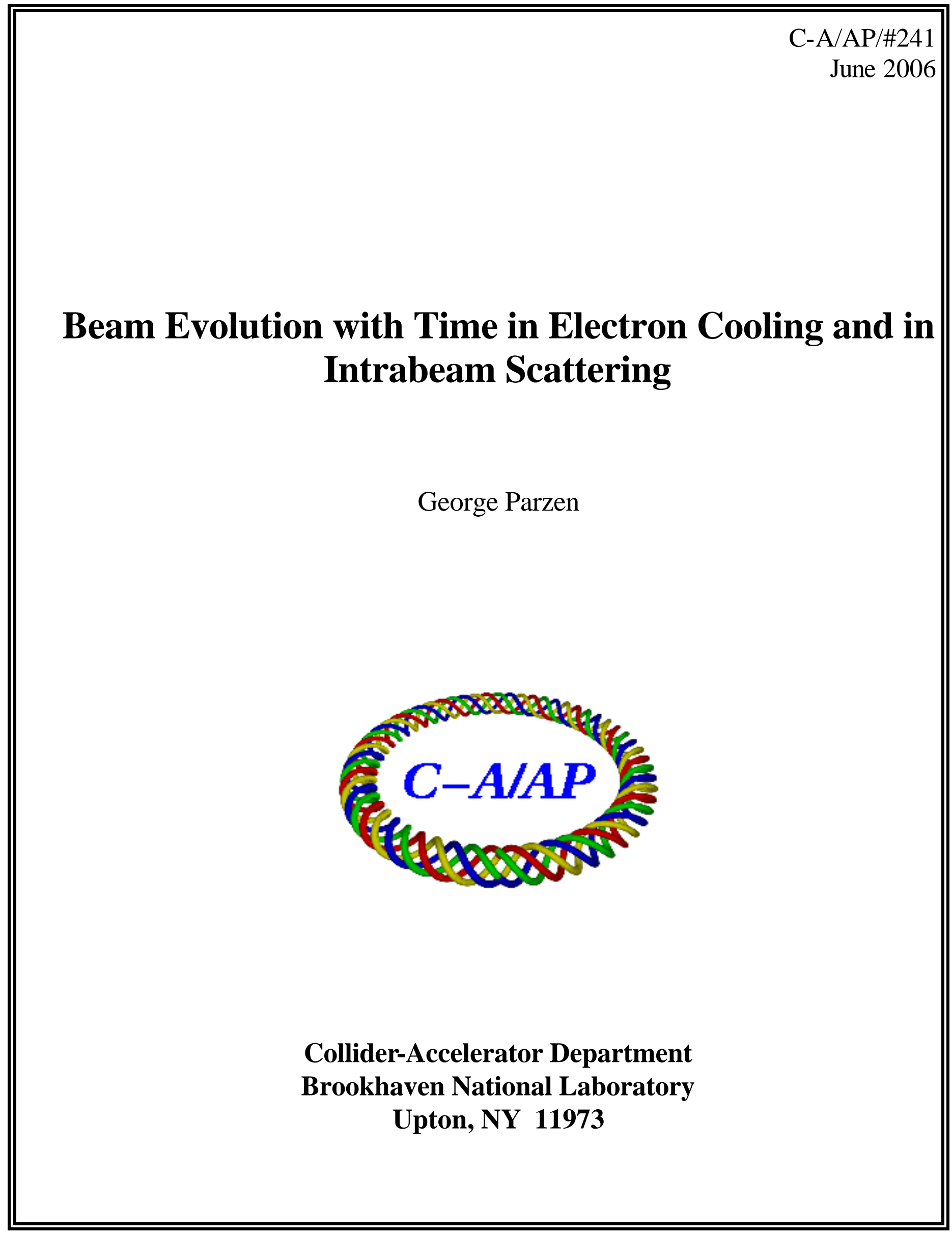




\title{
Beam evolution with time in electron cooling and in intrabeam scattering
}

\author{
George Parzen
}

June 12,2006

\begin{abstract}
Finding the evolution with time of the average emittances of an ion bunch in the presence of electron cooling and intrabeam scattering between the ions is a difficult problem to solve exactly. An approximate procedure is described for computing the evolution with time of the average emittances. This procedure is based on the treatment of electron cooling as an intrabeam scattering process [4] and the procedure proposed for finding the evolution with time of the average emittances of the ion bunch is similar to the one that has been used for the beam growth due to intrabeam scattering of the ions from each other.
\end{abstract}

\section{Introduction}

Finding the evolution with time of the average emittances of an ion bunch in the presence of electron cooling and intrabeam scattering between the ions is a difficult problem to solve exactly. An approximate procedure is described for computing the evolution with time of the average emittances. This procedure is based on the treatment of electron cooling as an intrabeam scattering process [4] and the procedure proposed for finding the evolution with time of the average emittances of the ion bunch is similar to the one that has been used for the beam growth due to intrabeam scattering of the ions from each other. 


\section{Intrabeam scattering growth of identical ions}

Intrabeam scattering theory as developed in [1] and then improved in [2] and [3] computes the growth rates of the average emittances of an ion bunch due to the scattering of identical ions from each other when the ions have a gaussian distribution. A difficulty in computing the growth of the average emittances with time arises because the ion distribution will probably not remain gaussian even if it starts as gaussian. The growth of the average emittances of the ion bunch with time has been computed by using a procedure which might be called the gaussian time integrator. In this procedure, the ion distribution, which may not be gaussian, is fitted with a gaussian distribution which has the same average emittance as the ion distribution. The growth rates of the average emittances of the ion distribution are then assumed to be the growth rates computed for the gaussian fit using the intrabeam scattering theory results. This procedure has been used to find the evolution with time of the average emittances of the ion bunch due to intrabeam scattering, and has been,to some extent,compared with measurements.

For an initial gaussian distribution, the growth rates found at $t=0$ are exact, except for the use of the truncated coulomb cross section to represent the interaction between the ions in the bunch. Thus the results for the beam evolution with time should be good for small enough $t$, then become less accurate at larger $t$ as the distribution starts to deviate from gaussian.

\section{Electron cooling as an intrabeam scattering process}

Recently [4] intrabeam scattering theory has been applied to electron cooling and expressions have been found for the cooling rates of the average ion emittances due to scattering between an ion bunch and an electron bunch which are moving with the same velocity and both having gaussian distributions. Using these results, one can find the evolution with time of the average emittances of the ion bunch due to intrabeam scattering and electron cooling, using the gaussian time integrator described above.The electron bunch and the ion bunch distributions are each fitted with gaussians having the same average emittances for each of the bunches. The eletron cooling is adjusted to cancel the growth due to intrabeam scattering between the ions. The BIG program has been modified to compute both intrabeam scat- 
tering growth rates and electron cooling cooling rates for the ion bunch,and the evolution with time of the average emittances of the ion bunch due to intrabeam scattering and electron cooling.

For an initial gaussian distribution, the growth rates found at $t=0$ are exact, except for the use of the truncated coulomb cross section. Thus the results for the beam evolution with time should be good for small enough $\mathrm{t}$, then become less accurate at larger $\mathrm{t}$ as the distribution starts to deviate from gaussian.

\section{Bigaussian time integrator}

The procedure using the gaussian time integrator could be improved by fitting the distributions with a bigaussian instead of a gaussian. The gaussian distribution requires 3 parameters while the bigaussian reqires 7 parameters. At present using the bigaussian has been worked out [5] for the case of intrabeam scattering of identical ions. These results would have to be extended to the case of the scattering of ions and electrons. Altogether, it would require quite a bit of work to use the bigaussian.

I thank Alexei Fedotov for his assistance.

\section{References}

1. A. Piwinski Proc. 9th Int. Conf. on High Energy Accelerators (1974) 405

2. J.D. Bjorken and S.K. Mtingwa, Part. Accel.13 (1983) 115

3. M. Martini CERN PS/84-9 (1984)

4. G. Parzen, BNL report,C-A/AP/No.232 (2006)

5. G. Parzen BNL report C-A/AP/N0.169 (2004) 